\title{
7 La grammaire de constructions : un atout pour la formation du traducteur?
}

\begin{abstract}
Résumé : Le présent article explore le potentiel d'application d'une théorie linguistique relativement jeune, la grammaire de constructions, pour la didactique de la traduction. C'est pourquoi nous commencerons par esquisser les grandes lignes et les principaux courants de la grammaire de constructions, parmi lesquels la grammaire radicale de constructions nous intéressera particulièrement. Nous verrons ensuite les différents arguments en faveur d'une application de cette modélisation dans la didactique de la traduction mais également, et plus généralement, en traductologie. Nous terminerons sur la nécessité d'une adaptation spécifique de cette théorie à la pratique de la traduction en considérant de manière plus précise les différents aspects de la dimension sémantique des constructions.
\end{abstract}

\section{Les grammaires de constructions}

Une question récurrente chez les auteurs d'introductions à la grammaire de constructions concerne l'emploi du pluriel ou du singulier : cette modélisation de la langue compte plusieurs courants dont les divergences sont parfois notables. Fischer \& Stefanowitsch (2008) choisissent de parler d'une «famille de théories » qui s'accordent toutes sur une conception générale du langage comme un inventaire de signes (paires signifiant-signifié). La description des structures langagières se résume alors à l'inventorisation de ces signes, appelés constructions, qui constituent le seul élément de base des systèmes linguistiques (traduction libre) ${ }^{1}$ (p. 3). Lakoff propose une définition du terme construction :

1 «Die Konstruktionsgrammatik ist derzeit (noch) keine einheitliche linguistische Theorie, sondern eher eine Familie von Theorie, die durch die Einsicht miteinander verbunden sind, dass die menschliche Sprache auf allen sprachlichen Ebenen aus Zeichen (also aus Form-Bedeutungspaaren) besteht. Folgerichtig gehen diese Ansätze davon aus, dass die Beschreibung der Strukturen einer Sprache ausschließlich auf der Beschreibung des Inventars solcher Zeichen - die als 〈Konstruktionen〉 bezeichnet werden - beruhen kann und sollte [...]» 
Each construction will be a form-meaning pair (F, M) where F is a set of conditions on syntactic and phonological form and $\mathrm{M}$ is a set of conditions on meaning and use (Lakoff, 1987: 467).

Croft insiste sur l'indifférenciation entre les constructions grammaticales et lexicales, propre aux grammaires de constructions, que nous appellerons « GrCons » selon François (2008), laissant deviner leur potentiel théorique pour l'étude et l'apprentissage de la traduction :

\footnotetext{
Grammatical constructions in construction grammar, like the lexicon in other syntactic theories, consist of pairings of form and meaning that are at least partially arbitrary. Even the most general syntactic constructions have corresponding general rules of semantic interpretation (Croft, 2001: 23).
}

L'idée qu'une construction grammaticale (par exemple la construction passif français) soit systématiquement décrite non seulement dans sa forme mais dans sa valeur sémantique représente un atout majeur pour l'étude et l'enseignement de la traduction, où la dimension sémantique joue un rôle central. C'est l'axiome essentiel de cette famille théorique : un continuum lexique-syntaxe, où la distinction entre des unités lexicales et des règles présidant à leur agencement disparaitt. Ainsi, les GrCons constituent en grande partie une rupture avec les théories linguistiques centrées sur la syntaxe. Nous retiendrons deux caractéristiques essentielles à notre réflexion : les GrCons sont non dérivationnelles et non modulaires.

\subsection{Non-dérivationnalité}

Le premier aspect qui distingue radicalement les GrCons d'autres théories est sans conteste le rejet de la dérivation. En s'affranchissant notamment des règles de transformation, on abandonne la description radiale du système linguistique où des exceptions d'usage, comme les expressions figées, gravitent autour d'un noyau de règles syntaxiques.

There are no derivations in CG [Construction grammar]. A candidate sentence is licensed as a sentence of the language if and only if there exists in the grammar of that language a set of constructions which can be combined in such a way to produce a representation of that sentence (Kay, 1997: 125).

Si le principe de dérivation ne s'applique pas, les constructions se combinent entre elles: une construction peut donc être composée de plusieurs constructions (c'est le cas pour toutes les constructions contenant plus d'un mot) et peut donc s'étudier à différents niveaux: affixes, désinences, lexèmes, proverbes, structures syntactiques abstraites (temps, modes), pourvu que ces structures 
soient associées à une signification précise. Dans la phrase Jean a donné un livre à Marie, on trouvera donc la construction sujet-prédicat ([SUJ PRED]), la construction ditransitive la construction passé composé, deux constructions groupe nominal ([NP $\left.\mathrm{N}_{\text {nom propre }}\right]$ et $\left[_{\mathrm{NP}}\right.$ Det $\left.\mathrm{N}\right]$ ), ainsi que les constructions lexicales Jean, Marie, livre, un et donn- (cf. Fischer \& Stefanowitsch, 2008 : 7). D’autre part, la phrase Jean a donné un livre à Marie peut également être considérée comme une construction et faire partie d'une construction plus complexe (un texte, par exemple).

\subsection{Les GrCons sont non modulaires}

La non-modularité des constructions est à la base des GrCons, les distinguant des autres théories génératives : forme et sens doivent impérativement être considérés dans leur relation réciproque, abandonnant l'idée que ces deux éléments sont mentalisés par le locuteur dans des modules différents.

In other words, constructions are like lexical items in the componential model: they link together idiosyncratic or arbitrary phonological, syntactic and semantic information. The difference between lexical items and constructions is that lexical items are substantive and ATOMIC (that is, minimal syntactic units), while constructions can be at least partially schematic and COMPLEX (consisting of more than one syntactic element) (Croft, 2001: 21).

Si ces deux caractéristiques (non-modularité, rejet de la dérivation) sont communes à toutes les GrCons, seuls certains courants présentent des atouts cruciaux pour l'étude de la traduction. Dans cette optique, nous nous concentrerons sur la variante de la GrCons qui nous semble la plus appropriée à une adaptation " pédagogique » en traductologie: la grammaire de constructions radicale. $^{2}$

2 Pour une liste très succincte des principales autres variantes, nous renverrons à Fillmore (1988) et Kay (1997) (Grammaire de constructions par unification, « École de Berkeley »), Langacker (1987) (Grammaire cognitive), Goldberg (1995) (Grammaire de constructions cognitive) et Lakoff (1987), Croft (2001) (Grammaire de constructions radicale). 


\section{La grammaire de constructions radicale (William Croft)}

[...] I propose that we discard the assumption that syntactic structures are made up of atomic primitives (language-universal or language-particular). Constructions, not categories and relations, are the basic primitive units of syntactic representation. [...] This is Radical Construction Grammar. (Croft, 2001: 60)

La grammaire de constructions radicale (RGrCons) est encore peu étudiée dans son application pratique, bien que son angle d'approche nous semble particulièrement adapté à la traduction et la traductologie : elle aborde expressément les différences entre les systèmes linguistiques. En effet, Croft rejette l'idée d'universaux syntaxiques du langage (Croft, 2001, p. 81) ${ }^{3}$, révoquant aussi la notion de catégories grammaticales valables pour toutes les langues et prônant une relativisation de la nomenclature afin de prendre en compte les différences interlinguistiques : la catégorie « sujet » devient « sujet français », similaire au « sujet allemand » mais pas identique.

La question de la compositionnalité des constructions est également traitée : pour Croft, non seulement la signification d'une construction complexe n'est pas la somme des significations des lexèmes et de règles combinatoires, mais elle est plus que la somme des significations des constructions qui la composent.

Enfin, les composantes d'une construction ne peuvent être définies que dans le cadre de la construction qu'elles constituent. Ce relativisme permet de modéliser la langue telle qu'elle est pratiquée. ${ }^{4}$

Les aspects les plus spécifiques de la RGrCons se résument donc comme suit :

- Il s'agit d'une modélisation de la langue basée sur l'usage uniquement.

- Elle prend en compte le spectre linguistique dans son intégralité, sans hiérarchisation centre-périphérie.

- Elle rejette les catégories syntaxiques universelles inter- comme intralinguistiques.

3 Ce qui lui a souvent été reproché par les universalistes. Toutefois, il précise : " There are universals of language, but not in syntactic structure taken by itself. The universals of language are found in semantic structure and in symbolic structure, that is, the mapping between linguistic function and linguistic form » (Croft, 2001: 81). La réalité pratique du traducteur, du traductologue et de l'étudiant en traduction étant toutefois précisément centrée sur les différences entre deux langues, la question des universaux linguistiques ou plutôt de leur nature apparaît ici comme secondaire.

4 Et l'on se détache ici du locuteur idéal de la grammaire générative et transformationnelle, ce qui permet une meilleure adéquation avec le travail de la linguistique appliquée. 
- Elle considère toute construction comme une paire signifiant-signifié, où le signifié se définit sur plusieurs niveaux : sémantique, pragmatique, fonctionnel, illocutoire, interactionnel, etc.

- Les constructions peuvent être polysémiques.

- Les composantes d'une construction ne peuvent être définies dans leur relation forme-sens que dans le contexte général de la construction qu'elles constituent. Il s'agit ici d'une approche non réductionniste où le niveau macroscopique détermine l'analyse au niveau microscopique.

\section{Potentiel de la grammaire de constructions radicale pour la traductologie et la formation du traducteur}

Les GrCons se comprennent en premier lieu comme une théorie linguistique. Il est question de décrire la langue (ou les langues) de la façon la plus complète et précise possible. Ainsi, leur utilisation en linguistique appliquée est encore relativement restreinte. Toutefois, la recherche dans le domaine de l'acquisition des langues s'appuie déjà de plus en plus souvent sur ces fondements (cf. Tomasello, 2008 ; Diessel, 2008 ; Haberzettl, 2008 ; Delorme Benites, 2011). La traductologie et l'enseignement de la traduction, en revanche, ne mentionnent presque jamais les GrCons.

Le potentiel de la RGrCons dans la formation du traducteur se dessine cependant en filigrane à travers nombre de théories actuelles en matière de traduction. C'est pourquoi un rapide tour d'horizon des différentes approches permet de mieux imaginer la place que pourrait y occuper la RGrCons.

\subsection{En théorie : comment concilier traductologie et grammaire de constructions}

Nous prendrons ici comme référence l'Introduction à la traductologie de Guidère (2008) à titre d'exemple. Premier constat : il n'aborde qu'épisodiquement la question des modèles linguistiques généraux sous-tendant aux théories de la traduction. Il souligne expressément le besoin de distanciation des traductologues par rapport à la linguistique générale comme seule référence explicative, citant une " insuffisance des outils conceptuels de la linguistique » et « le besoin de faire appel à d'autres disciplines pour appréhender le phénomène traductologique » 
constaté par Pergnier (1978). Ce « refus de la linguistique » était également exprimé par l’École de Paris de la manière suivante :

\begin{abstract}
Quels qu'aient été les mérites de la linguistique [...] elle ne peut prétendre expliquer la complexité de la traduction [...] les linguistiques structurales et génératives ont été mues par ce qui nous apparaît aujourd'hui comme un complexe d'infériorité à l'égard des sciences exactes. Elles se sont efforcées de façon quasi obsessionnelle de réifier la langue. En en faisant un objet observable de façon objective, elles se voulaient scientifiques. En se limitant au mesurable, quantifiable et prévisible, elles ont sacrifié l'essentiel du langage : son emploi en situation par un individu pensant (Lederer, 1994: 92).
\end{abstract}

Sans nier que l'étude de la traduction est bien plus qu'une sous-catégorie de la linguistique et qu'elle est assurément interdisciplinaire, nous voyons dans la critique de la linguistique générale par Lederer et Pergnier une critique d'une approche, dont il est aujourd'hui possible de s'affranchir. En effet, la RGrCons part de l'usage réel de la langue pour l'inventorier. D'une énonciation de règles normative, on passe à une description contextualisée et spécifique pour chaque langue. Il serait donc possible de réintégrer cette approche plus moderne et, finalement, plus pragmatique dans l'initiation aux réflexions traductologiques, comme une modélisation de la langue facilitant l'étude de la traduction et de ses mécanismes.

On trouve par ailleurs des éléments proches de la logique sous-jacente aux GrCons dans diverses théories de la traduction. La théorie interprétative de l'École de Paris (Lederer, 1994) et ses trois phases de traduction (interprétation, déverbalisation, réexpression), se prête aisément à un parallèle avec la définition-même des constructions, dans lesquelles le sens (objet central de la déverbalisation) est constamment présent. De même, le concept largement connu et employé d' « équivalences » (Catford, 1965) s'accorde parfaitement avec un modèle de la langue où toute différence de forme implique systématiquement une différence de sens, et ce même au sein d'une même langue. Enfin, les « unités de traduction » présentées par Vinay et Darbelnet (1958), souvent discutées (entre autres en regard de la différenciation entre unités de structure et unités de sens) ${ }^{5}$ et même reformulées par Larose (1989) en « traductèmes » rappellent fortement les constructions.

Ainsi, bien qu'une modélisation aussi récente que la RGrCons ne soit pas encore intégrée aux réflexions traductologiques, les différentes approches abordent parfois les faits linguistiques de manière similaire. Szymańska résume bien la portée de cette modélisation linguistique pour l'ensemble des approches théoriques de la traduction :

5 Une dichotomie à laquelle l'application de la RGrCons pourrait peut-être mettre un terme ? 
$\mathrm{CxG}$ is inherently functional and usage-based, being maximalist in the aim of describing the totality of the communicative conventions available to the language user. It has a potential to address the issues of pragmatics, text structure, text interpretation and the influence of culture-bound knowledge on the use of language, which makes it capable of acknowledging the insights of the pragmatic turn and the cultural turn in translation studies (Szymańska, 2011: 223).

\subsection{En pratique : un exemple pour l'enseignement de la traduction}

Comme vu précédemment, la RGrCons abandonne la conception de la langue comme un noyau d'éléments « corrects » ou « normaux » autour duquel gravitent toutes sortes de variétés (sociolectes, idiolectes...) et de variations. Toutes les productions acceptées par un locuteur sont donc incluses dans cette description, ce qui permet à l'enseignant d'aborder de façon systématique des problèmes de traduction fréquents dans la pratique professionnelle, notamment pour la localisation de slogans (publicitaires...) ou, par exemple en traduction audio-visuelle ou de jeux vidéo, des constructions sociolectiques (langage enfantin, langue des jeunes...). Un exemple tiré du langage des jeunes serait la traduction vers l'allemand de la construction entièrement spécifiée :

[La tête de moi !]

L'étudiant se retrouve confronté à deux problèmes :

- Cette phrase n'est pas correcte grammaticalement (dans un système normatif traditionnel).

- Son sens n'est pas (ou plus) déductible du sens de ses différentes composantes.

Cette expression ne figurant pas dans les dictionnaires, il sera difficile d'en trouver le sens au moyen des recherches habituelles privilégiées par les étudiants. Une explication basée sur la RGrCons serait:

la construction

[La tête de moi !]

est une spécification de la construction

[Ø la tête de $\mathrm{N}$ !]

elle-même héritée de 
[Je le jure sur la tête de $\mathrm{N}$ ] / assurance ou insistance sur la véracité d'un fait.

On notera l'addition de la construction

[ ! ] / le fait est difficile à croire, le fait est peu commun.

Enfin, la construction entièrement spécifiée [La tête de moi !] porte les aspects :

un fait difficile à croire est vrai (sens),

le locuteur appartient au groupe social « jeune Français de classe sociale défavorisée ».

Cette analyse amène l'étudiant vers la recherche d'une catégorie sociale équivalente dans la langue cible puis, au sein de ce sociolecte, d'une construction courante exprimant « un fait difficile à croire » ou « la surprise » (par extension). Ainsi, l'étudiant pourrait finalement proposer en allemand :

[Krass, Alter!]

ou autre exclamation similaire dont la forme, hormis le point d'exclamation, n'a rien à voir avec la construction française de départ. Si le traducteur expérimenté parvient souvent directement à une solution comparable, le chemin de l'étudiant et de son professeur pour arriver à cette compétence est bien souvent long et difficile à expliciter de façon systématique. La RGrCons offre un cadre explicatif très approprié à un meilleur accompagnement des expériences de l'étudiant.

Un second aspect de la RGrCons intéressant pour l'enseignement de la traduction réside dans l'absence de constructions synonymes : toute différence dans la forme impliquera une différence sémantique ou pragmatique. On balaie ainsi toute idée d'équivalences parfaites entre deux constructions de langues différentes, soulignant l'essence même de la traduction, et il devient plus clair pour l'étudiant que la recherche monolingue de "synonymes » ou de "paraphrases » dans ses efforts de (re)formulation ne peut s'arrêter à la consultation d'un dictionnaire des synonymes, qui ne tient bien souvent pas (ou pas assez) compte de l'usage. Nous reprendrons ici l'argumentation de Szymańska en faveur d'une explication constructionnelle de la notion de gains et pertes en traduction :

When considered jointly, [form and meaning] highlight and explain the fact that in translating we are often restricted by the resources of the target language, which may cluster various properties differently than the resources of the source language, which were used in the source text for certain communicative purposes (Szymańska, 2011: 220).

Elle expose l'impératif fréquent de prioriser les aspects regroupés dans la catégorie « sens » (pragmatiques, sémantiques, fonctionnels, illocutoires, etc.) d'une construction-source afin de choisir une construction-cible de la manière la plus 
adéquate possible au contexte, prenant en compte l'abandon de certains aspects considérés comme moins prioritaires. Puisque sens et forme sont des composantes inhérentes aux constructions, la construction-cible choisie pourra, en outre, comprendre des aspects supplémentaires par rapport à la constructionsource : c'est la notion bien connue de gains et de pertes.

Jusqu'ici, cette problématique était facilement explicable au niveau lexical. Les constructions plus syntaxiques et les constructions textuelles peuvent ainsi être abordées selon la même logique: la question, par exemple, du vouvoiement ou du tutoiement dans un texte injonctif (les modes d'emploi, classiques de la traduction), s’inscrit dans un cadre théorique constructionnel permettant une réflexion approfondie englobant tous les aspects des différentes constructions d'adresse (vouvoiement et tutoiement français, Siezen et Duzen allemands, [You] anglais, etc.) pour motiver le choix final de la construction-cible.

Ainsi, la RGrCons permet d'aborder de façon systématisée et uniforme les problèmes de traduction à tous les niveaux : lexème, expressions, phrases et textes. Résultat : la notion-même de construction dissout le rapport souvent trop fort chez l'étudiant entre sens et catégorie grammaticale. Il s'agit ici de prendre du recul par rapport, par exemple, à une phrase, et d'en contempler non plus les divers mots mais les ou la construction(s) pour en accéder au sens. Ce sens étant partie intégrante des constructions à traduire, l'étudiant devra chercher dans la langue d'arrivée des constructions dont ce même sens est également partie intégrante. Cette façon de procéder s'affranchit des catégories grammaticales traditionnelles présentes dans le texte de départ et dont l'étudiant débutant a du mal à se détacher.

In very general terms, constructional thinking about language is highly relevant to translation since it presents text interpretation and production as relying on recognizing functional wholes rather than assembling small elements. It is well-known that in translation the failure to recognize functional wholes often leads to disastrous effects. (Szymańska, 2011: 220)

\section{Conclusion et perspectives}

Les postulats avancés par la RGrCons offrent, en regard des préoccupations traductologiques contemporaines, un cadre de linguistique générale en adéquation avec la compréhension actuelle des processus de traduction. Nous y voyons une modélisation qui, se détachant des concepts traditionnels de catégories grammaticales universelles, crée certes une rupture avec les pratiques de description linguistique habituelles dans les cursus de traduction, mais permet de relativiser les 
faits linguistiques pour une meilleure comparaison entre les différents systèmes et d'uniformiser l'approche réflexive des différents niveaux d'analyse (lexique, syntaxe, texte) dans une logique d'intégration.

À l'heure actuelle, il manque une mise en pratique adaptée aux réflexions traductologiques et didactiques : les travaux constructionnels traitent encore essentiellement de questions de linguistique générale. Il serait cependant essentiel pour une bonne intégration à la formation du traducteur de se pencher plus sur les différentes dimensions du « sens » des constructions. En effet, si la plupart des GrCons s'accordent sur une définition très vaste de la " sémantique » d'une construction, une approche concrète et structurée serait la bienvenue afin d'expliciter les aspects sémantiques, pragmatiques, illocutoires, métalinguistiques, interactionnels, culturels, etc.

D’autre part, le potentiel didactique de la RGrCons présente l'avantage d'amener les traducteurs débutants de manière méthodique aux solutions de traduction que choisiraient probablement des traducteurs expérimentés. Est-ce alors l'expérience qui forme un inventaire d'équivalences de constructions chez le traducteur? Une telle hypothèse rejoint les études constructionnelles menées sur l'acquisition des langues chez l'enfant et l'adulte (Diessel, 2008 et Tomasello, 2008). Il serait ici intéressant d'étudier comment se forme l'inventaire des constructions équivalentes dans deux langues chez le traducteur et dans quelle mesure il est possible d'optimiser ce processus chez l'étudiant. Nous conclurons en insistant sur les innombrables voies que peut ouvrir l'intégration d'une théorie linguistique générale telle que la grammaire de constructions radicale à la recherche et à l'enseignement de la traduction, dans l'esprit d'un dialogue constructif entre linguistique générale et linguistique appliquée.

\section{Références bibliographiques}

Catford, J. C. (1965). A linguistic theory of translation; an essay in applied linguistics. London: Oxford University Press.

Croft, W. (2001). Radical construction grammar: Syntactic theory in typological perspective. Oxford: Oxford University Press.

Delorme Benites, A. (2011). Konstruktionen im mündlichen Diskurs von ausländischen Doktoranden in der alläglichen Wissenschaftssprache: Eine konstruktionsgrammatisch angelegte Fallstudie. Siegen: Universitätsbibliothek der Universität Siegen.

Diessel, H. (2008). Komplexe Konstruktionen im Erstspracherwerb. Dans Fischer, K., et Stefanowitsch, A. (dir.). Konstruktionsgrammatik I ( $2^{\mathrm{e}}$ éd., pp. 39-54). Tübingen: Stauffenburg.

Fillmore, C. J. (1988). The mechanisms of "Construction grammar". Annual meeting of the Berkeley Linguistics Society 14, 35. doi:10.3765 / bls.v14i0.1794 
Fischer, K., et Stefanowitsch, A. (2008). Konstruktionsgrammatik: Ein Überblick. Dans Fischer, K., et Stefanowitsch, A. (dir.). Konstruktionsgrammatik I ( $2^{\mathrm{e}}$ éd., pp. 3-17). Tübingen: Stauffenburg.

François, J. (2008). Les grammaires de construction, un bâtiment ouvert aux quatre vents (Cahier du CRISCO n²6). Caen. Repéré à : http://www.crisco.unicaen.fr/IMG/pdf/Cahier_ CRISCO_26_FRANCOIS1.pdf le 25.01.2017.

Goldberg, A. E. (1995). Constructions: a construction grammar approach to argument structure. Chicago: University of Chicago Press.

Guidère, M. (2008). Introduction à la traductologie: Penser la traduction: Hier, aujourd'hui, demain. Bruxelles: De Boeck.

Haberzettl, S. (2008). Konstruktionen im Zweitspracherwerb. Dans Fischer, K., et Stefanowitsch, A. (dir). Konstruktionsgrammatik I ( $2^{\mathrm{e}}$ éd., pp. 55-77). Tübingen: Stauffenburg.

Kay, P. (1997). Construction grammar. In Kay, P. (dir.). Words and the grammar of context (pp. 123-131). Stanford, CA: CSLI Publications. Également repéré à : http://www1.icsi. berkeley.edu/ kay/bcg/cg_define.html.

Lakoff, G. (1987). Women, fire, and dangerous things: What categories reveal about the mind. Chicago: University of Chicago Press.

Langacker, R. W. (1987). Foundations of cognitive grammar. Stanford. CA: Stanford University Press.

Larose, R. (1989). Théories contemporaines de la traduction. Québec: Université du Québec.

Lederer, M. (1994). La traduction aujourd'hui: le modèle interprétatif. Paris: Hachette.

Pergnier, M. (1978). Les fondements sociolinguistiques de la traduction. Paris: Honoré Champion.

Szymańska, I. (2011). Construction Grammar as a framework for describing translation. Dans Pawlak, M. \& Bielak, J. (dir.). New perspectives in language, discourse and translation studies (Second language learning and teaching) (pp. 215-225). Berlin-Heidelberg: Springer.

Tomasello, M. (2008). Konstruktionsgrammatik und früher Erstspracherwerb. Dans Fischer, K. et Stefanowitsch, A. (dir.) Konstruktionsgrammatik I ( $2^{\mathrm{e}}$ éd., pp. 19-37). Tübingen: Stauffenburg.

Vinay, J., \& Darbelnet, J. (1958/1977). Stylistique comparée du français et de l'anglais: méthode de traduction. Paris: Didier. 